\title{
Afecto y matemáticas. Diseño de una entrevista para acceder a los sentimientos de alumnos adolescentes
}

\author{
Patricia Pérez-Tyteca, Universidad de Valencia (España) \\ Javier Monje, Universidad de Alicante (España) \\ Enrique Castro, Universidad de Granada (España)
}

Recibido el 30 de Abril de 2013; aceptado el 16 de Septiembre de 2013

\begin{abstract}
Afecto y matemáticas. Diseño de una entrevista para acceder a los sentimientos de alumnos adolescentes

Resumen

En este documento presentamos un instrumento que hemos diseñado con el objeto de obtener información sobre la ansiedad matemática y la autoconfianza en matemáticas de alumnos que realizan el paso de la educación secundaria a la educación universitaria así como su relación con el género y la elección de titulaciones. Se trata de una entrevista con la que buscamos superar las dificultades de comunicación que se generan cuando se pregunta directamente por sus sentimientos a los adolescentes para lo que hemos recurrido a técnicas proyectivas. En este artículo mostramos además los resultados obtenidos de su aplicación, que permiten valorar su idoneidad.
\end{abstract}

Palabras clave. Ansiedad matemática, autoconfianza, género, entrevista, carreras

Afeto e matemática. Projeto de uma entrevista para acessar os sentimentos de estudantes adolescentes

\section{Resumo}

Neste artigo, apresentamos uma ferramenta que nós projetamos para o propósito de obter informações sobre a ansiedade matemática e matemática autoconfiança nos alunos, tendo a etapa do ensino secundário para o ensino superior e sua relação com gênero e escolha grau. Esta é uma entrevista em que buscamos superar as dificuldades de comunicação que são gerados quando os seus sentimentos perguntar por adolescentes para o que usaram técnicas projetivas. Mostramos também os resultados da sua aplicação, que permitem avaliar a sua adequação.

Palavras chave. Ansiedade matemática, auto-confiança, sexo, entrevista, carreira

Affect and mathematics. Design of an interview to access the feelings of teenagers students

Abstract

In this paper we present a tool we designed for the purpose of obtaining information about math anxiety and math self-confidence in students taking the step of higher education to university education and its relationship to gender and degree choice. This is an interview in which we seek to overcome

Para citar: Pérez-Tyteca, P., Monje, J., \& Castro, E. (2013). Afecto y matemáticas. Diseño de una entrevista para acceder a los sentimientos de alumnos adolescentes. Avances de Investigación en Educación Matemática, 4, 65-82.

(C) Sociedad Española de Investigación en Educación Matemática (SEIEM). www.seiem.es 
communication difficulties that are generated when your feelings ask by teens for what we have used projective techniques. We show also the results of its application, which allow to assess their suitability.

Key words. Math anxiety, self-confidence, gender, interview, degrees

Affection et les mathématiques. Conception d'une interview pour accéder aux sentiments des élèves adolescents

Résumé

Dans cet article, nous présentons un outil que nous avons conçu dans le but d'obtenir des informations sur l'angoisse des mathématiques et de mathématiques confiance en soi chez les élèves qui prennent l'étape de l'enseignement secondaire à l'enseignement supérieur et de sa relation à l'égalité et le choix du degré. Il s'agit d'une interview dans laquelle nous cherchons à surmonter les difficultés de communication qui sont générés lors de vos sentiments demandent par les adolescents pour ce que nous avons utilisé des techniques projectives. Nous montrons également les résultats de son application, qui permettent d'évaluer leur pertinence.

Paroles clés. L'angoisse des mathématiques, la confiance en soi, le sexe, interview, université

El diseño y aplicación del instrumento que vamos a mostrar en este artículo se enmarca dentro de un proyecto más amplio que contempla, además de esta fase cualitativa, un estudio cuantitativo basado en un estudio descriptivo y el posterior diseño y evaluación de un modelo causal de ecuaciones estructurales. En la fase cuantitativa se atiende a diversas variables afectivas, demográficas y académicas. De entre ellas, vamos a centrarnos en la ansiedad matemática, la autoconfianza, el género y la elección de titulaciones universitarias.

A este respecto, los resultados que arroja el estudio cuantitativo indican que dentro del conjunto de estudiantes que realizan el paso de la educación secundaria a la universitaria, existe correlación negativa entre su ansiedad matemática y su autoconfianza, que se producen en ambos constructos diferencias significativas de género a favor de los hombres y que tienen efecto sobre la elección de la titulación universitaria que realizan.

Teniendo en cuenta estos resultados y siguiendo las recomendaciones, cada vez más unánimes, de la comunidad investigadora que defienden la combinación de métodos cuantitativos y cualitativos en el estudio del afecto, nos hemos marcado como objetivo diseñar y aplicar una entrevista que nos permita profundizar en la ansiedad matemática y la autoconfianza de los estudiantes que terminan su educación secundaria y arroje luz sobre cómo estos constructos influyen en la toma de decisiones relativa a la elección de titulación universitaria. Las características de esta entrevista y la valoración de su eficacia a partir de los resultados derivados de su aplicación se detallan en el presente documento.

\section{Marco Teórico}

Hoy en día, la importancia que tiene el afecto dentro del proceso de enseñanza y aprendizaje de las matemáticas es innegable. Los investigadores de nuestro país no son ajenos a esta realidad, y aunque se llevan realizado cuantiosos trabajos sobre afecto- como queda reflejado en la revisión realizada por Gómez-Chacón (2010)- el número de trabajos realizados recientemente en torno a este tópico va en aumento 
(Caballero, 2013; Cauich \& Hidalgo, 2012; Estrada, Bazán \& Aparicio, 2013; López \& Alsina, 2013; Martins, Nascimento \& Estrada, 2012; Mellado, Blanco, Borrachero \& Cárdenas, 2012; Montoro \& Gil, 2012; Palacios, Hidalgo, Maroto \& Ortega, 2013; Rodríguez del Tío, Hidalgo \& Palacios, 2012; Sánchez, 2013). Uno de los aspectos afectivos que posee más tradición dentro de nuestro campo de investigación es la ansiedad matemática.

En este trabajo consideramos la ansiedad matemática como un estado afectivo caracterizado por la ausencia de confort que puede experimentar un individuo en situaciones relacionadas con las matemáticas, tanto de su vida cotidiana como académica, y que se manifiesta mediante un sistema de respuestas que engloban una serie de "síntomas", como son: tensión, nervios, preocupación, inquietud, irritabilidad, impaciencia, confusión, miedo y bloqueo mental.

El estudio de este constructo se inició hace más de 40 años y, sin embargo, sigue siendo un tema de plena actualidad. Prueba de ello es su inclusión en el estudio PISA 2003 implementado en 40 países, en el que se ha comprobado que una gran proporción de los alumnos de 15 años evaluados manifiestan sentimientos de inseguridad y estrés emocional cuando se enfrentan a las matemáticas. Según este estudio, los alumnos que sienten ansiedad cuando estudian matemáticas tienden a no interesarse en su estudio ni disfrutar con ellas, siendo ésta una relación consistente en todos los países que constituye pues un patrón de relaciones universal. Dada la fuerte prevalencia de la ansiedad entre los estudiantes, es importante proseguir investigando en esta área (OCDE, 2004).

Un efecto indirecto de la ansiedad matemática que pasa por el bajo rendimiento, es evitar asistir a cursos relacionados con las matemáticas, ya que para evitar el sentimiento de ansiedad, los afectados evitan el cursar la materia (Tobias \& Weissbrod, 1980), lo que condiciona posteriormente el tipo de carrera universitaria que se puede tomar (Seaman, 1999). Así lo señala también Ashcraft (2002), para quien "los individuos con niveles altos de ansiedad matemática se caracterizan por su gran tendencia a evitar las matemáticas, cosa que a la larga debilita sus competencias matemáticas y les impide tomar ciertas trayectorias en su carrera" (p. 181).

Además de estas importantes consecuencias de la ansiedad matemática, el efecto que el género tiene sobre ella es un aspecto que ha sido ampliamente estudiado y se ha comprobado que existe una evidencia de que las mujeres sufren más ansiedad matemática que los hombres (Fennema \& Sherman, 1976; Gil, Blanco \& Guerrero, 2006; Lafferty, 1994; Vest, 1998; Wigfield \& Meece, 1988; Worley, 1997).

La ansiedad matemática no es una respuesta afectiva que se presente aislada, sino que, está vinculada a otros factores afectivos. Uno de ellos es la autoconfianza (que definimos como la confianza que un sujeto tiene en sí mismo como aprendiz de matemáticas), que aparece en numerosas investigaciones (Fennema \& Sherman, 1978; Gonske, 2002; Isiksal, Curran, Koc \& Askun, 2009; Swars, 2004; Wigfield \& Meece, 1988) negativamente correlacionada con ella.

De manera consistente con esta relación, en los trabajos sobre el tema, la autoconfianza ha sido frecuentemente relacionada con la participación por parte de los estudiantes en asignaturas, cursos y carreras que requieren matemáticas (Fox, 1976; McLeod, 1992) y se ha comprobado que las mujeres poseen menor confianza que los hombres en sus habilidades matemáticas (Hannula \& Malmivuori, 1997; Marsh \& 
Ayotte, 2003; Nurmi, Hannula, Maijala \& Pehkonen, 2003; Pierce, Stacey \& Barkatsas, 2007).

Por este motivo en nuestro trabajo consideramos ambos constructos (ansiedad matemática y autoconfianza), que encuadramos dentro del marco teórico propuesto por McLeod (1989), que considera el dominio afectivo como "un extenso rango de estados de ánimo que son generalmente considerados como algo diferente de la pura cognición, e incluye como componentes específicos las creencias, las actitudes y las emociones" (p. 245).

Como indican Hart (1989) y Evans (2000), algunos investigadores en educación matemática consideran la ansiedad matemática como una actitud. Sin embargo, McLeod (1992) apunta que la utilización de este término "no parece adecuado para describir algunos sentimientos más intensos que los estudiantes exhiben en las clases de matemáticas" (p. 576). Por su parte, los psicólogos sociales categorizan la ansiedad matemática como una emoción, siendo considerada una respuesta visceral. La visión de la ansiedad matemática como una actitud conlleva considerarla una respuesta afectiva más "fría" y de menor intensidad y puede identificarse con la ansiedad-rasgo) frente a la caracterización de la ansiedad matemática como una respuesta intensa ante experiencias matemáticas específicas (que se identifica con la ansiedad-estado). A este respecto, Hannula (2011), además de tener en cuenta la ansiedad-estado, ubica la ansiedad-rasgo como una disposición emocional, que condiciona una serie de reacciones emocionales típicas (worry, fear) ante situaciones típicas de aprendizaje.

De acuerdo con esto, nosotros defendemos tener en cuenta ambas caracterizaciones (ansiedad como actitud y ansiedad como emoción) en el estudio de la ansiedad matemática, ya que "aunque las emociones de un estudiante pueden fluctuar y cambiar rápidamente durante la resolución de un problema, los estudiantes también tienen patrones emocionales muy estables en el tiempo" (Hannula, 2011, p. $45)$.

La autoconfianza, por su parte, la ubicamos dentro de las creencias y, tomando la clasificación que realiza McLeod (1992) de las mismas, la consideramos una creencia sobre uno mismo como aprendiz de matemáticas.

\section{Metodología}

El diseño metodológico empleado en este trabajo consiste en un estudio de casos instrumental múltiple. Este tipo de investigación es generalmente descriptiva y se lleva a cabo mediante el estudio en profundidad de una cantidad limitada de sujetos escogidos de manera intencionada para ilustrar un determinado problema.

La técnica de recogida de datos que hemos empleado consiste en una entrevista semiestructurada cuyas características detallamos en el siguiente epígrafe.

\section{El instrumento}

Como indican Hernández, Fernández-Collado \& Baptista (2006), "las entrevistas, como herramientas para recolectar datos cualitativos, se emplean cuando el problema de estudio no se puede observar o es muy difícil hacerlo por ética o complejidad y permiten obtener información personal detallada" (p. 598). 
Así, consideramos adecuado la realización de entrevistas para profundizar en aspectos como los relativos al dominio afectivo. Concretamente, en el estudio de la ansiedad matemática, aunque la mayoría de los trabajos utilizan técnicas meramente cuantitativas, existen también investigadores que han realizado estudios cualitativos, que prácticamente en su totalidad son entrevistas (Bekdemir, 2010; Gavira, 2008; Gresham, 2007; Johnson, 2006; Jones, 2006; Uusimaki \& Nason, 2004). Las hemos revisado y hemos comprobado que todas ellas preguntan directamente a los sujetos por sus sentimientos. De este modo, dado que en nuestro trabajo pretendemos romper la barrera inicial que puede existir en los adolescentes para hablar con naturalidad y confianza de lo que sienten, hemos decidido diseñar nuestra propia entrevista de modo que vayamos aproximándonos a las reacciones afectivas de los estudiantes de una manera menos brusca.

La entrevista que hemos diseñado es semiestructurada, es decir, el entrevistador (y en este caso investigador) crea una guía de temas que abordar pero tiene la libertad de incluir preguntas adicionales con el fin de obtener mayor información de los temas tratados.

\section{Focos de atención de la entrevista}

Como ya hemos apuntado, previo al diseño de la entrevista, hemos llevado a cabo un estudio cuantitativo que profundiza en constructos como la ansiedad matemática, la autoconfianza o la utilidad y que aborda las relaciones existentes entre diferentes variables afectivas, académicas y demográficas. La intención de profundizar en estos constructos y arrojar luz sobre la naturaleza de estas relaciones es la que sirve de guía para la confección de la entrevista, y determina sus focos de atención, que son los que se describen a continuación.

Utilidad para la vida-elección de titulación: Al entrevistar a los estudiantes seleccionados exploraremos cuán útiles consideran las matemáticas tanto para su vida cotidiana como para su vida laboral teniendo en cuenta si la carrera que han escogido posee asignaturas relacionadas con la materia.

Género-elección de titulación: Con respecto a esta relación, y teniendo en cuenta que en el análisis descriptivo de los datos realizado en la primera fase de nuestro trabajo se observa que el número de mujeres matriculadas en titulaciones con gran orientación matemática es bajo y sin embargo existen otras titulaciones prácticamente femeninas, pretendemos obtener información sobre las opiniones de los sujetos al respecto. Es decir, nos interesa saber si ellos consideran que existen titulaciones más apropiadas para uno u otro sexo y cuál es su punto de vista sobre las razones que provocan los desequilibrios en cuanto a género presentes en algunas carreras universitarias.

Ansiedad- rendimiento: Otro de los focos de atención de las entrevistas será la relación entre ansiedad matemática y rendimiento en la asignatura. A este respecto indagaremos en la percepción por parte de los entrevistados de la existencia o no de dicha relación y su aplicación a su caso particular.

Género-ansiedad: Hemos comprobado en el análisis de las escalas cumplimentadas por alumnos de bachillerato, que existen diferencias de género siendo las mujeres las que presentan mayor ansiedad matemática. En la fase de entrevistas participarán sujetos de ambos sexos que cumplen la condición de que los hombres, aun rindiendo menos que sus compañeras en matemáticas, reportan en la escala 
menores niveles de ansiedad. Así podremos profundizar en los sentimientos de cada uno para poder valorar hasta qué punto se corresponden con lo expresado en el cuestionario,

Rendimiento-elección de titulación: Esta relación la analizaremos desde la perspectiva de aquellos estudiantes seleccionados cuya nota media en matemáticas en los dos primeros trimestres del curso es baja (suspenso). De ellos hay tres que pese a ello desean estudiar titulaciones con gran orientación matemática y otro que desea estudiar alguna titulación de nivel 2 aunque no especifica cuál. Nos interesa obtener información sobre por qué consideran que obtienen calificaciones bajas y, en unos casos cómo se plantean mejorar dichas calificaciones de cara a poder optar por la titulación escogida, y en el otro hasta qué punto dicha nota influye en la elección final de la carrera.

Autoconfianza-ansiedad: Tanto en los resultados recogidos en la literatura como en los análisis descriptivo y causal realizados en este trabajo queda patente la relación existente entre ansiedad matemática y autoconfianza. Mediante las entrevistas pretendemos comprobar si los estudiantes que demuestran ansiedad matemática también poseen baja autoconfianza y cuál es la naturaleza de esta relación.

Por medio de las entrevistas profundizaremos también en la naturaleza de las variables objeto de nuestro estudio, que son las que se detallan a continuación.

Ansiedad matemática: Un foco esencial de la entrevista es el referido a indagar en la ansiedad matemática de los sujetos seleccionados. Nos interesa conocer cuáles son sus sentimientos hacia la asignatura y en qué momentos los experimentan. Así, obtendremos información sobre la ansiedad hacia las matemáticas en general (un día normal de clase, cuando se sale a la pizarra, al realizar taras en casa, etc.), la ansiedad hacia los exámenes y la ansiedad hacia los problemas, que son los indicadores de la ansiedad matemática recogidos en el modelo causal.

Autoconfianza: Es interesante profundizar en la capacidad percibida por parte de los estudiantes y en su concepción de que existen personas que sirven para las matemáticas y otras que no. Además pretendemos conocer si se sienten inseguros con la materia y en qué situaciones se manifiesta dicha inseguridad.

Elección de titulación: En el modelo causal planteado en este trabajo se relaciona la elección de la titulación con aspectos como la ansiedad matemática, el género o el rendimiento, pero nos interesa obtener información sobre qué otros factores intervienen en la decisión de la carrera universitaria que se va a cursar. Por este motivo preguntaremos a los entrevistados qué valores han tenido en cuenta a la hora de decidirse.

\section{Uso de viñeta}

En la entrevista, la función del entrevistador es fundamentalmente la de servir de catalizador de una expresión exhaustiva de los sentimientos y opiniones del sujeto. Para esto se debe crear una atmósfera facilitadora en la que el sujeto halle libertad para expresarse.

Para crear esta atmósfera recurrimos a realizar preguntas proyectivas. Las preguntas proyectivas llevan implícita la proyección de la íntima forma de pensar o sentir del entrevistado. Están basadas en las técnicas proyectivas de obtención de datos. Éstas son técnicas que motivan al entrevistado para que proyecte sus 
motivaciones, creencias, actitudes, sentimientos ocultos respecto a los temas de interés, de modo que el entrevistado proyecta su propia opinión en algo ajeno a él (por ejemplo, una tercera persona). Siempre resulta más sencillo poner en boca de otro lo que uno siente, sobre todo si tiene gran carga emotiva. La utilización de estas técnicas, además tienen un gran valor para propiciar un ambiente agradable que genere un clima cómodo y relajado donde los participantes se desinhiban, permitiendo de este modo adquirir la información deseada sin que el entrevistado se sienta comprometido directamente.

De manera frecuente, en la aplicación de técnicas proyectivas se estimula la imaginación a través de imágenes. Como indica Fischman (2006), "la incorporación de la cultura visual en la agenda de la investigación y el agrandamiento de la caja de herramientas metodológicas para los investigadores en educación, deberían ser considerados un desafío" (p. 252). Es más, este mismo autor afirma que basándose en su propia experiencia puede asegurar que el uso de imágenes (fotos, viñetas o dibujos), provoca la obtención de más respuestas por parte de los sujetos y facilita el acceso a información relacionada con temas que de otro modo evitan.

Por tanto, consideramos adecuado utilizar preguntas proyectivas en la entrevista que estén basadas en imágenes visuales. De este modo pretendemos "atravesar" la barrera defensiva que probablemente proteja a los estudiantes de hablar de sus propios sentimientos.

De entre las imágenes visuales, Twerski (1997), defiende el uso de las viñetas para abordar asuntos relacionados con la psicología ya que el uso de caricaturas hace que el paciente se sienta identificado de algún modo con el personaje pero a la vez suficientemente alejado de él para observar a distancia la situación evitando sentirse amenazado y cohibido.

Así pues, en nuestro trabajo hemos elegido esta forma gráfica- las viñetas- ya que consideramos que éstas nos dan la oportunidad de contar historias de fácil lectura y comprensión, que resulten atractivas para los estudiantes, y que estén protagonizadas por personajes con los que puedan identificarse. De este modo, partiendo de la historia relatada en las viñetas animaremos a los entrevistados a hablar de las situaciones en ellas relatadas, accediendo de manera paulatina a sus propios sentimientos.

\section{Guion de la entrevista}

La entrevista empieza con una presentación por parte de la entrevistadorainvestigadora que verifica algunos de los datos demográficos proporcionados por el sujeto al rellenar las escalas (rendimiento, estudios que se pretenden cursar...). A continuación se procede a abordar el primer bloque temático, que pretende extraer información de cuán útiles consideran los sujetos que son las matemáticas y cuyo guión es el siguiente:

Entrevistadora Bien, en primer lugar me gustaría saber si consideras que las matemáticas son útiles para ti. ¿En qué áreas y en qué sentido crees que te van a ayudar?

El segundo bloque temático está introducido por una historieta contada en viñetas (véase Figura 1) y pretende extraer información sobre las experiencias de ansiedad 
matemática experimentadas por los sujetos y sobre las partes de la disciplina que causan más respuestas afectivas (exámenes, problemas, ejercicios). El guión de este bloque es el que sigue:

Entrevistadora Ahora quiero que veas esta historieta en la que aparecen Luís y Ana y me digas qué opinas, ¿vale?

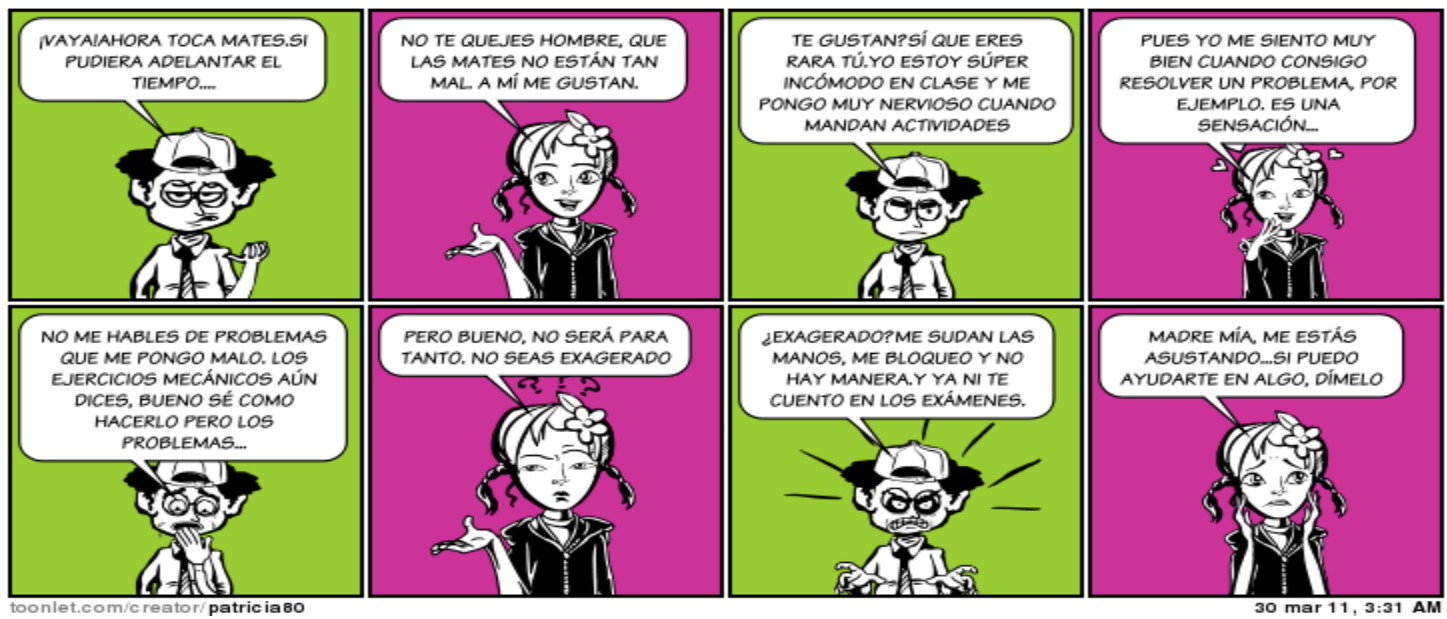

Figura 1. Primer conjunto de viñetas de la entrevista.

Entrevistadora Bueno, ¿qué opinas de lo que pasa en la historieta? ¿Te sientes de algún modo identificado/a con algún personaje? ¿Por qué?

En el tercer bloque temático se pretende profundizar en la capacidad que los entrevistados consideran tener para hacer matemáticas y en los sentimientos de inseguridad al enfrentarse a la materia. Este bloque también va introducido por una serie de viñetas (véase Figura 2) que dan continuación a la historia relatada en el bloque anterior. Su guión se presenta a continuación:

Entrevistadora Bien, sigamos con la historia

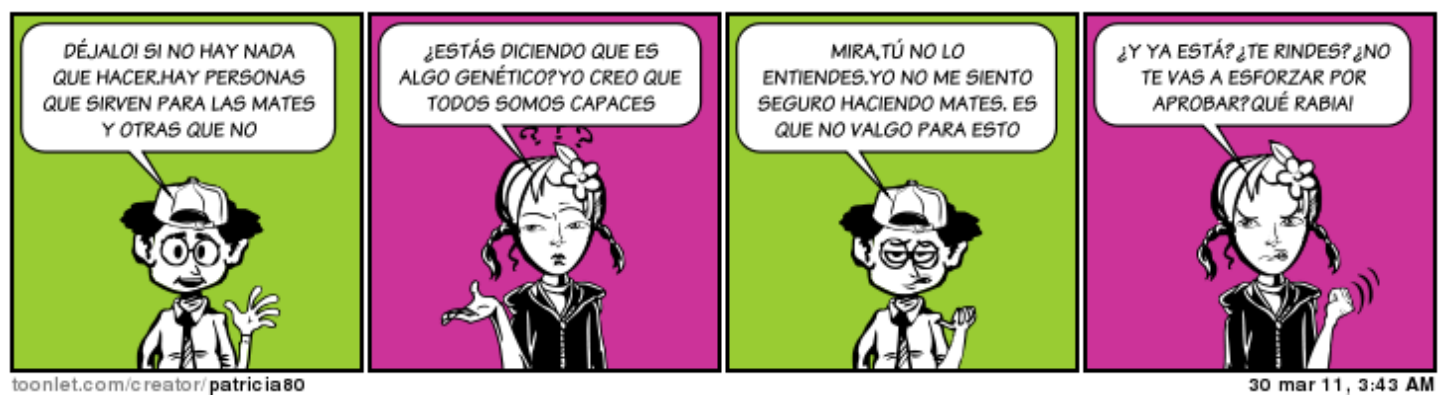

Figura 2. Segundo conjunto de viñetas de la entrevista.

Entrevistadora ¿Estás de acuerdo con que existen personas que no sirven para las matemáticas y otras que sí? ¿De qué crees que depende? ¿Qué características tienen las personas que no sirven? Luis nombra la inseguridad ¿estás de acuerdo? ¿Qué puedes decirme al respecto sobre ti mismo/a? 
El final de la historieta, presentado en la Figura 3, introduce el cuarto bloque temático que pretende extraer información sobre el modo en que la ansiedad matemática influye en el rendimiento y sobre cómo lleva a la evitación de las matemáticas por parte de los sujetos ansiosos. El guión correspondiente a este bloque es el siguiente:

\section{Entrevistadora Vamos a ver ahora como termina la historia}

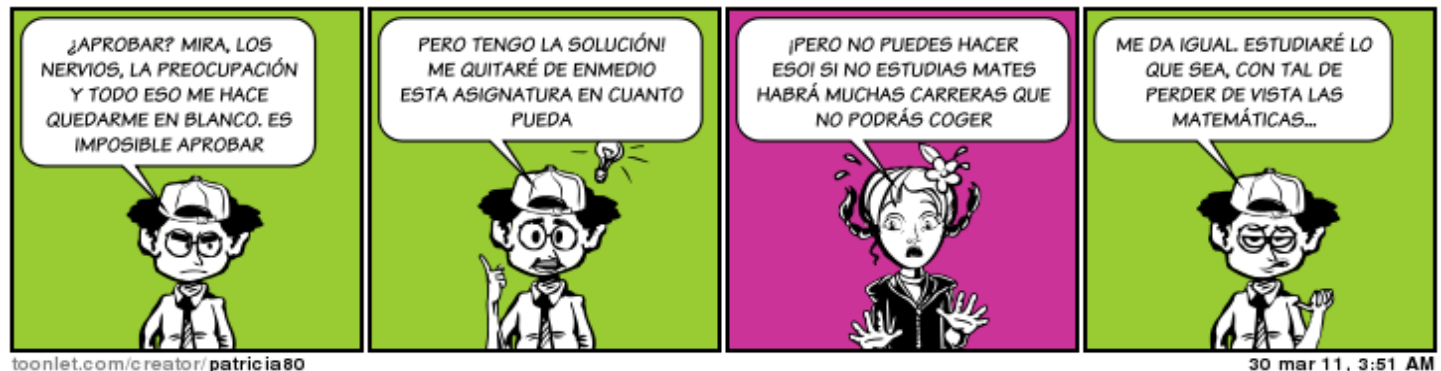

Figura 3. Tercer conjunto de viñetas de la entrevista.

Entrevistadora ¿Qué opinas de lo que dicen Luís y Ana? ¿Has vivido tú algo similar? $¿$ ¿Crees que los sentimientos hacia las matemáticas influyen en las notas que se sacan? ¿Y crees que esto condiciona a algunos estudiantes a elegir cierto tipo de estudios y otros no? ¿Qué puedes decirme de tu caso al respecto?

El bloque número cinco pretende obtener datos sobre el proceso de toma de decisión respecto a la titulación universitaria que se va a cursar, y sobre la existencia de prejuicios sociales que identifican ciertos estudios con un género determinado. El guion de este bloque es el que se detalla a continuación:

Entrevistadora Muy bien, pues ya para ir terminando me gustaría que me cuentes qué te ha llevado a elegir la titulación que has elegido estudiar. ¿Cómo fue el proceso de decisión?

¿Crees que existen carreras más apropiadas para hombres que para mujeres o viceversa? Existen carreras con mayoría masculina, como ingenierías o arquitectura y otras con mayoría femenina, como magisterio o enfermería ¿A qué crees que se debe?

Para finalizar, la investigadora-entrevistadora agradece la colaboración del entrevistado y le invita a despejar cualquier duda que pueda tener sobre el proceso de entrevista.

\section{Participantes}

Para la selección de los sujetos a entrevistar se administraron las escalas de Ansiedad Matemática, Autoconfianza y Utilidad de las matemáticas de FennemaSherman (1976) a los estudiantes de segundo de bachillerato de dos centros 
educativos de la ciudad de Granada: uno público y uno privado. Además se les preguntó por su rendimiento en matemáticas y por la titulación que habían decidido escoger. Estos estudiantes estaban en sus últimos días de clase y por tanto ya tenían decidido qué estudios superiores desean cursar. Los datos obtenidos se han analizado con el fin de obtener información del perfil afectivo y académico de cada sujeto.

A la hora de elegir a los alumnos que vamos a entrevistar nos interesa seleccionar a aquellos alumnos que posean ansiedad alta o muy alta y comprobar cómo ésta influye en el tipo de titulación elegida.

Consideramos conveniente elegir también aquellos casos en los que un alto grado de ansiedad va acompañado de un alto rendimiento con el fin de obtener información sobre cómo estos sujetos afrontan su ansiedad al enfrentarse a las tareas matemáticas y consiguen desempeñarlas de manera satisfactoria.

Los estudiantes con un alto grado de ansiedad y una alta autoconfianza también son de interés, ya que nuestro modelo pronostica una correlación negativa entre ambos constructos. Es decir, una alta ansiedad está vinculada a una baja autoconfianza.

Los resultados del estudio cuantitativo que hemos llevado a cabo previamente a la fase del trabajo que estamos relatando en este artículo muestran que aquellos sujetos que deciden estudiar carreras claramente orientadas a las matemáticas poseen un rendimiento alto en la materia y la consideran muy útil para el desarrollo de su vida. Por tanto, en caso de que algún participante de bachillerato no cumpla esta premisa, consideramos interesante seleccionarlo para la fase de entrevistas.

Por último, con el fin de buscar información que arroje luz sobre las diferencias de género en ansiedad matemática (que también se producen en este grupo de alumnos aun teniendo mayor rendimiento las chicas que los chicos), hemos decidido incluir en las entrevistas a dos hombres y dos mujeres, habiendo reportado los primeros menor ansiedad que sus compañeras aun teniendo peor rendimiento.

Bajo los criterios establecidos para la selección de participantes, hemos elegido a 7 estudiantes para que participen en la fase de entrevistas. Las características de cada uno de ellos se reflejan en la Tabla 1.

Tabla 1. Características de los sujetos seleccionados para las entrevistas.

\begin{tabular}{|c|c|c|}
\hline Código & Género & Características \\
\hline 3 & $\mathrm{M}$ & $\begin{array}{l}\text { Ansiedad alta, autoconfianza baja, titulación con orientación } \\
\text { matemática baja, participa en el estudio de género }\end{array}$ \\
\hline 14 y 43 & $\mathrm{H}$ & $\begin{array}{l}\text { Rendimiento bajo, titulación con orientación matemática alta, } \\
\text { participa en el estudio de género }\end{array}$ \\
\hline 18 & M & Rendimiento alto, ansiedad media \\
\hline $\mathrm{C} 7$ y $\mathrm{C} 9$ & M & $\begin{array}{l}\text { Ansiedad alta, rendimiento bajo, están decidiendo qué titulación } \\
\text { escoger }\end{array}$ \\
\hline $\mathrm{C} 1$ & M & $\begin{array}{l}\text { Ansiedad alta, rendimiento bajo, titulación con orientación } \\
\text { matemática alta }\end{array}$ \\
\hline
\end{tabular}

\section{Proceso de aplicación}

La realización de las entrevistas se llevó a cabo en horario académico pero siempre procurando que los estudiantes no tuvieran que faltar a sus clases. La duración de las mismas fue de aproximadamente 20 minutos cada una y se realizaron 
en la sala de visitas del centro educativo o en un aula vacía. En primer lugar se procedió a la recogida de los consentimientos y asentimientos informados, para dar paso al comienzo de la entrevista, que fue grabada en vídeo para facilitar su posterior análisis. La investigadora procuró en todo momento que el alumno entrevistado se sintiera cómodo y relajado y se ofreció a aclarar cualquier duda que le surgiera.

\section{Análisis de los datos}

El análisis de los datos obtenidos se realizó mediante la transcripción y análisis de las repuestas dadas por los estudiantes. Para extraer información y establecer relaciones sobre cada uno de los aspectos estudiados, agrupamos las respuestas de los sujetos seleccionados para tal fin y creamos una serie de categorías basadas, bien en la orientación de sus respuestas (p.e. en el caso de la idoneidad de hombres y mujeres para las matemáticas) o bien en la naturaleza de las respuestas afectivas manifestadas, estableciendo para estos casos diferentes grados de intensidad de las mismas. Así, a modo de ejemplo, diremos que un sujeto posee ansiedad matemática alta si declara sentirse muy nervioso, preocupado o tenso cuando se enfrenta a la materia. Estos alumnos suelen sentirse identificados con el personaje de Luis, y si sus sentimientos no son tan extremos lo especifican, lo que nos indica una reacción menos negativa y nos permite identificar cuál es su grado de ansiedad.

\section{Resultados}

Los resultados más significativos obtenidos a partir de las respuestas de los sujetos que hemos entrevistado son los siguientes.

La gran mayoría de los entrevistados no considera de gran utilidad las matemáticas para su vida cotidiana. En cuanto a la utilidad para su vida académica y laboral se observan diferencias entre aquellos alumnos que han elegido carreras sin matemáticas (que no las consideran útiles en absoluto).y los que se han decantado por alguna titulación que sí contempla la materia en su plan de estudios (que sí las consideran útiles). Dentro de este último grupo los estudiantes que se han decantado por titulaciones más técnicas tienen más clara la utilidad de la materia para su vida académica que aquellos que han escogido una titulación con menor orientación matemática.

En el caso de la relación rendimiento-ansiedad hemos entrevistado a tres sujetos con bajo rendimiento que pese a ello han escogido titulaciones técnicas. Dos de los entrevistados (que reportan en la escala niveles bajos de ansiedad) admiten haberse esforzado poco en el estudio de las matemáticas y afirman ser capaces de superar la materia si se esfuerzan más mientras que la tercera participante (que reporta valores altos de ansiedad) no confía en sus posibilidades y confiesa sentirse preocupada por las matemáticas que deberá cursar en caso de poder entrar en la carrera, pero se deja llevar por la ilusión de estudiar algo que realmente le gusta. Así, vemos que aunque el rendimiento influye en la elección de la titulación, la ansiedad matemática también juega un papel importante ya que los que la poseen van minando su seguridad y confianza hasta convencerse de su incapacidad para hacer frente a titulaciones orientadas a la materia.

En cuanto a los estereotipos relativos a la idoneidad de uno u otro género para desarrollar determinadas profesiones, todos los participantes consideran igualmente 
capaces tanto a hombres como a mujeres para cursar cualquier titulación pero la mayoría admite que en nuestra sociedad todavía existen ciertos prejuicios que encasillan a cada género en un tipo de ocupaciones.

Además, hemos indagado qué factores influyen en la elección de la titulación universitaria y hemos descubierto que, aunque la gran mayoría basa esta decisión en sus gustos personales, la situación económica y social por la que atraviesa nuestro país en estos momentos provoca que los estudiantes también tengan en cuenta cuáles son las oportunidades de trabajo que les brinda cada carrera.

En cuanto a las respuestas de ansiedad que experimentan los participantes cuando hacen matemáticas, los resultados que hemos obtenido son los siguientes.

Las respuestas dadas por los entrevistados muestran que todos ellos, a excepción de uno, poseen cierto grado de ansiedad. De manera general, las situaciones de examen son las que más ansiedad provocan. Este resultado coincide con el obtenido en la fase cuantitativa de nuestro trabajo (fase previa a la relatada en el presente documento) y también con investigaciones como la de Sánchez (2013).

Aterrizando en las tareas que se suelen trabajar en el aula, los problemas provocan una serie de respuestas de ansiedad que los ejercicios de mera aplicación de algoritmos no provocan. Dentro de estas respuestas de ansiedad se encuentra el rechazo inicial que provoca que el alumno se tense por el simple hecho de tener que hacer un problema sin haber evaluado ni siquiera su dificultad o el proceso a seguir para su resolución. En cuanto al ambiente de aula, hemos comprobado que los alumnos que se sienten incómodos o tensos en el transcurso de una clase normal lo están debido a su temor por ser sacados a la pizarra, aunque este temor puede variar en función del tipo de profesor que se tenga.

Además, hemos comprobado que ninguno de los entrevistados conoce técnicas de auto-control que pueda aplicar para tratar las respuestas de ansiedad surgidas en los exámenes. En este punto se aprecia la importancia de trabajos como el de Caballero, Guerrero, Blanco y Piedehierro (2009) y Caballero (2013), que realizan una implementación para el aula basada en la resolución de problemas con el fin de dotar a los futuros docentes de técnicas de afrontamiento y control emocional que mejoren sus estados de ansiedad y bloqueo.

En cuanto a la relación ansiedad-rendimiento hemos verificado que, a excepción del alumno que no reporta ansiedad, todos los entrevistados creen que los sentimientos negativos hacia las matemáticas influyen en las calificaciones en la materia y que si ellos consiguieran vencer lo que sienten sus notas mejorarían. Estos resultados confirman la correlación negativa que ya anunciaba Reyes (1984) en su revisión de la literatura que vincula la ansiedad matemática con el rendimiento en la materia.

Por otro lado, las respuestas de los entrevistados reflejan que aquellos estudiantes que han manifestado experiencias de ansiedad en sus respuestas poseen una baja capacidad percibida para afrontar las matemáticas y se sienten inseguros ante ella a diferencia del sujeto que no ha mostrado ansiedad, que dice sentirse seguro ante la materia y no duda de su capacidad para enfrentarse a ella. Estos resultados constatan la fuerte correlación negativa existente entre ansiedad matemática, fenómeno que puede observarse en trabajos precedentes tan diversos como los realizados por Fennema \& Sherman (1978), Gonske (2002) e Isiksal et al. (2009). 
De este modo, hemos comprobado cómo aquellos estudiantes con ansiedad matemática y baja autoconfianza están preocupados por las matemáticas que deberán estudiar en la universidad, aun habiendo escogido carreras con bajo perfil matemático.

Uno de los factores más estudiado con referencia a la ansiedad matemática es el género. Esto es debido a que, desde el inicio de su estudio hasta la actualidad, se han hallado diferencias de género por las que las mujeres reportan mayor ansiedad que los hombres. Son variados los motivos que se han barajado para explicar este fenómeno pero consideramos que algunos de ellos (como el trato diferenciado que el profesor ofrece a niños y niñas en el aula o la creencia de que las mujeres no poseen capacidad matemática) no tienen cabida en los tiempos que corren. Por tanto, en la búsqueda de una teoría que explique por qué siguen dándose diferencias a día de hoy, hemos encontrado una hipótesis que puede encajar y que es defendida por autores como Reyes (1984), Martin (1994) o Perina (2002). Esta hipótesis contempla la posibilidad de que las mujeres tiendan a explicitar más sus experiencias de ansiedad al rellenar un instrumento de auto-reporte que los hombres. De este modo, ante sentimientos similares, las mujeres asignarían puntuaciones más altas a los ítems de la escala.

Comprobar si esta premisa se da en un aula de bachillerato ha sido uno de nuestros objetivos al planear las entrevistas. Para ello hemos comprobado si existen sujetos que hayan obtenido puntuaciones diferentes en la escala pero demuestren experimentar sensaciones similares Con respecto a ello, las respuestas a la entrevista apuntan a que los sujetos C9 y 14 poseen un nivel de ansiedad similar y sin embargo la alumna C9 obtuvo en la escala una puntuación que indica que posee un nivel alto de ansiedad mientras que el estudiante 14 reflejaba en la escala que su nivel de ansiedad es bajo.

Por tanto aquí tenemos un caso en el que, ante sensaciones similares, se otorgan puntuaciones diferentes al rellenar el instrumento de auto-reporte dependiendo del sexo del sujeto.

\section{Conclusiones}

De los resultados obtenidos al realizar la entrevista se desprende la gran influencia que ejercen variables afectivas, como la ansiedad matemática y la autoconfianza, en la vida académica de los estudiantes.

Este resultado constata la importancia de complementar los estudios cuantitativos con una parte cualitativa, que ayude a entender la naturaleza y características de las respuestas afectivas de los sujetos. Para poder realizar de manera efectiva esta combinación debemos contar con instrumentos cualitativos que sean sensibles a los obstáculos asociados al estudio de un tema tan delicado como es el afecto.

En el caso de la entrevista es fundamental conseguir que el entrevistado no se sienta cohibido y sea capaz de exhibir sin temor sus sentimientos.

En este sentido la inclusión de historietas contadas en viñetas en la entrevista ha demostrado ser un elemento facilitador a la hora de extraer información.

Así pues, consideramos que éste es un paso en la buena dirección que abre la puerta a una nueva forma de comunicación en el estudio del afecto que pasa de la pregunta directa a la aproximación del problema por medio de una situación ajena y atractiva para el entrevistado que consigue derribar las barreras que pueden existir al hablar de los propios sentimientos. 


\section{Referencias}

Ashcraft, M. H. (2002). Math anxiety: Personal, educational, and cognitive consequences. Current Directions in Psychological Science, 11(5), 181-185.

Bekdemir, M. (2010). The pre-service teachers' mathematics anxiety related to depth of negative experiences in mathematics classroom while they were students. Educational Studies in Mathematics, Online First. Recuperado el 6 de mayo del 2012 de http://www.springerlink.com/content/3184p45718207m46/fulltext.pdf.

Caballero, A. (2013). Diseño, aplicación y evaluación de un programa de intervención en control emocional y resolución de problemas matemáticos para maestros en formación inicial. (Tesis doctoral no publicada). Universidad de Extremadura, Badajoz, España.

Caballero, A., Guerrero, E., Blanco, L. J., \& Piedehierro, A. (2009). Resolución de problemas de matemáticas y control emocional. En M. J. González, M. T. González \& J. Murillo (Eds.), Investigación en Educación Matemática XIII, 151-160.

Cauich, A. R., \& Hidalgo, S. (2012). Cognición, afectividad y diversidad. Una propuesta de determinación de perfiles matemáticos en los estudiantes. En A. Estepa, Á. Contreras, J. Deulofeu, M. C. Penalva, F. J. García, \& L. Ordóñez (Eds.), Investigación en Educación Matemática XVI, 177-185.

Estrada, A., Bazán, E., \& Aparicio, A. (2013). Evaluación de las propiedades psicométricas de una escala de actitudes hacia la estadística en profesores. Avances de Investigación en Educación Matemática, 3, 5-23.

Evans, J. (2000). Adults' Mathematical Thinking and Emotions. Londres: Routledge Falmer.

Fennema, E., \& Sherman, J. A. (1976). Fennema-Sherman mathematics attitude scales. Instruments designed to measure attitudes toward the learning of mathematics by males and females. JSAS Catalog of Selected Documents of Psychology, 6(31). (Ms. No. 1225).

Fennema, E., \& Sherman, J. A. (1978). Sex-related differences in mathematics achievement and related factors: A further study. Journal for Research in Mathematics Education, 9 , 189-203.

Fischman, G. (2006). Aprendiendo a sonreir aprendiendo a ser normal. En I. Dussel \& D. Gutierrez (Comp.), Educar la Mirada. Políticas y Pedagogías de la imagen. (pp. 235254). Buenos Aires: Manantial.

Fox, L. H. (1976). The effects of sex role socialization on mathematics participation and achievement. Disponible en la base de datos Education Resources Information Center (ERIC, ED 142867).

Gavira, N. (2008). Análisis de las causas del alto índice de reprobación en la asignatura de matemáticas en el bachillerato. Trabajo presentado en el Primer Congreso Internacional de Educación Media Superior y Superior, Ciudad de México, México.

Gil, N., Blanco, L., \& Guerrero, E. (2006). El papel de la afectividad en la resolución de problemas matemáticos. Revista de Educación, 340, 551-569.

Gómez-Chacón, I.M. (2010). Tendencias actuales en investigación en matemáticas y afecto. En M.M. Moreno, A. Estrada, J. Carrillo \& T.A. Sierra, (Eds.), Investigación en Educación Matemática XIV (pp. 121-140). Lleida: SEIEM.

Gonske, T. L. (2002). Relationships among mathematics anxiety, beliefs about the nature of mathematics and the learning of mathematics, and students'learning approaches in nontraditional. (Tesis doctoral). University of Northern Colorado, Greeley, EE.UU. 
Gresham, G. (2007). A study of mathematics anxiety in pre-service teachers. Early Childhood Education Journal, 35(2), 181-188.

Hannula, M. S. (2011). The structure and dynamics of affect in mathematical thinking and learning. En M. Pytlak, T. Rowland \& E. Swoboda (Eds.), Proceedings of the Seventh Congress of the European Society for Research in Mathematics Education, 34-60.

Hannula, M. S., \& Malmivuori, M. L. (1997). Gender differences an their relation to mathematics classroom context. En E. Pehkonen (Ed.), Proceedings of the $21^{\text {th }}$ Conference of the International Group for the Psychology of Mathematics Education, 3, $33-40$.

Hart, L. E. (1989). Describing the affective domain: Saying what we mean. En D.B. McLeod, \& V.M. Adams (Eds.), Affect and mathematical problem solving: A new perspective (pp. 37-48). New York: Springer-Verlag.

Hernández, R., Fernández-Collado, C., \& Baptista, P. (2006). Metodología de la Investigación. México: Mc Graw Hill.

Isiksal, M., Curran, J. M., Koc, Y., \& Askun, C. S. (2009). Mathematics anxiety and mathematical self-concept: considerations in preparing elementary-school teachers. Social Behavior and Personality, 37(5), 631-643.

Johnson, C. E. (2006). Attitude or anxiety: Mathematics disposition of high school algebra I students. (Tesis de maestría). Wichita State University, Kansas, EE.UU. Recuperado el 5 de noviembre del 2012 http://soardev.wichita.edu/bitstream/handle/10057/650/t06087.pdf;jsessionid=1CBC4A0215097E1 $\underline{\mathrm{B} 8 \mathrm{CDB} 584 \mathrm{AE} 001770 \mathrm{D} \text { ? sequence }=1}$

Jones, V. O. (2006). Cognitive processes during problem solving of middle school students with different levels of mathematics anxiety and self-esteem: case studies. (Tesis doctoral). Florida State University, Tallahassee, EE.UU.

Lafferty, J. S. (1994). The links among mathematics text, students' achievement, and students' mathematics anxiety: A comparison of the incremental development and traditional texts. (Tesis doctoral). Widener University, Chester, EE.UU.

López, P., \& Alsina, A. (2013). ¿Cómo influyen las creencias sobre uno mismo en relación a las matemáticas? Una aproximación al papel de la identidad matemática en futuros maestros. En A. Berciano, G. Gutiérrez, A. Estepa \& N. Climent (Eds.), Investigación en Educación Matemática XVII, 345-353.

Marsh, H. W., \& Ayotte, V. (2003). Do multiple dimensions of self-concept become more differentiated with age? The differential distinctiveness hypothesis. Journal of Educational Psychology, 95(4), 687-706.

Martin, C. L. (1994). A discriminant study of memories, attitudes and beliefs that identify individuals who report feelings of math anxiety. (Tesis doctoral). Adler School of Professional Psychology.

Martins, J.A., Nascimento, M.M., \& Estrada, A. (2012). Caminando: las actitudes hacia la estadistica en estudiantes universitarios en el norte de Portugal. En A. Estepa, Á. Contreras, J. Deulofeu, M. C. Penalva, F. J. García \& L. Ordóñez (Eds.), Investigación en Educación Matemática XVI, 375-383.

Mellado, V., Blanco, L.J., Borrachero, A.B., \& Cárdenas, J. (Ed.) (2012). Las emociones en la enseñanza y el aprendizaje de las ciencias y de las matemáticas. Badajoz: Grupo DEPROFE.

McLeod, D. (1989). Beliefs, attitudes, and emotions: New views of affect in mathematics education. En D.B. McLeod \& V.M. Adams (Eds.), Affect and Mathematical Problem Solving: A New Perspective (pp. 245-258). New York: Springer-Verlag. 
McLeod, D. (1992). Research on affect in mathematics education: A reconceptualization. En D.A. Grows (Ed), Handbook of Research on Mathematics Teaching and Learning (pp. 575-596). New York: Macmillan Publishing Company.

Montoro, A.B., \& Gil, F. (2012). Elaboración y aplicación de un instrumento para medir experiencias de flujo. En A. Estepa, Á. Contreras, J. Deulofeu, M. C. Penalva, F. J. García \& L. Ordóñez (Eds.), Investigación en Educación Matemática XVI, 397-406.

Nurmi, A., Hannula, M. S., Maijala, H., \& Pehkonen, E. (2003). On pupils' self-confidence in mathematics: gender comparisons. En N. A. Pateman, B. J. Dougherty \& J. Zilliox (Eds.) Proceedings of the 27th Conference of the International Group for the Psychology of Mathematics Education, 3, 453-460.

OCDE (2004). Informe PISA 2003: Aprender para el Mundo del Mañana. España: Santillana.

Palacios, A., Hidalgo, S., Maroto, A., \& Ortega, T. (2013). Causas y consecuencias de la ansiedad matemática mediante un modelo de ecuaciones estructurales. Enseñanza de las Ciencias, 31(2), 93-111.

Perina, K. (2002). The sum of all fears. Psychology Today, 35(6), 19-19.

Pierce, R., Stacey, K., \& Barkatsas, A. N. (2007). A scale for monitoring students' attitudes to learning mathematics with technology. Computers and Education, 48(2), 285-300.

Reyes, L. H. (1984). Affective variables and mathematics education. The Elementary School Journal, 84(5), 558-581.

Rodríguez del Tío, P., Hidalgo, S., \& Palacios, A. (2012). La ansiedad matemática en alumnos de Grados en Estadistica. En A. Estepa, Á. Contreras, J. Deulofeu, M. C. Penalva, F. J. García \& L. Ordóñez (Eds.), Investigación en Educación Matemática XVI, 469-478.

Sánchez, J. (2013). Actitudes hacia las matemáticas de los futuros maestros de Educación Primaria. (Tesis doctoral no publicada). Universidad de Granada, Granada, España.

Seaman, C. R. (1999). I've got a secret: Math Anxiety. Philosophy of Mathematics Education Journal, 11. Recuperado el 10 de septiembre de 2013 en http://people.exeter.ac.uk/PErnest/pome11/art16.htm

Swars, S. (2004). Mathematics teaching efficacy beliefs of elementary preservice teachers and their relationship to mathematics anxiety. (Tesis doctoral). University of Alabama, Tuscaloosa, EE.UU.

Tobias, S., \& Weissbrod, C. (1980). Anxiety and mathematics: An update. Harvard Educational Review, 50(1), 63-70.

Twerski, A. (1997). ¿Cuándo Empezarán a Ir Bien las Cosas?: Un Recorrido Terapéutico por los Altibajos de la Vida (con la Ayuda de Charlie Brown y sus Amigos). Paidos Ibérica: Barcelona.

Uusimaki, L., \& Nason, R. (2004). Causes underlying pre-service teachers' negative beliefs and anxieties about mathematics. En M. Høines \& A. Fuglestad (Eds.), Proceedings of the 28th annual conference of the International Group for the Psychology of Mathematics Education, 4, 369-376.

Vest, B. (1998). Algebra I as an eighth-grade course: An examination of attitudes, mathematics anxiety, motivation, and achievement. (Tesis doctoral). University of Alabama, Tuscaloosa, EE.UU.

Wigfield, A., \& Meece, J. L. (1988). Math anxiety in elementary and secondary school students. Journal of Educational Psychology, 80, 210-216.

Worley, A. (1997). Mathematics anxiety in fourth, fifth, and sixth grade students: Origins and correlates. (Tesis doctoral). Temple University, Philadelphia, EE.UU. 


\section{Referencias a los autores}

Patricia Pérez-Tyteca, Universidad de Valencia (España).patricia.perez-tyteca@uv.es Javier Monje, Universidad de Alicante (España). monjejavier@ua.es.

Enrique Castro, Universidad de Granada (España). ecastro@ugr.es 


\title{
Affect and mathematics. Design of an interview to access the feelings of teenagers students
}

\author{
Patricia Pérez-Tyteca, Universidad de Valencia (España) \\ Javier Monje, Universidad de Alicante (España) \\ Enrique Castro, Universidad de Granada (España)
}

In this paper we present the qualitative phase of our work, which is part of a larger project that explores several affective, demographic and academic variables. In this phase, we designed and applied a semi-structured interview to obtain information on math anxiety and self-confidence in mathematics of students who are making the transition from secondary school to university and the relation with gender and career choice. The interview uses projective techniques, vignettes specifically.

The interviews were conducted with 7 students who were in the last year of "bachillerato" (two pre-university courses) from two schools in Granada (Spain): one state and one private. It was one of the last days of class and the students had already decided which university studies they wanted to pursue.

The results indicate the great influence that affective variables such as math anxiety and self-confidence have on degree choice. We have shown how negative affective experiences encountered when taking mathematics classes create a desire to avoid them, which determines the future of students who suffer from this anxiety. As a consequence, students rule out certain degrees only because mathematics is involved, depriving themselves of the opportunity to prepare for a profession that they may have ability to perform well.

Due to the characteristics of the interview, in this study we were able to verify that when faced with the same feelings, women tend to give a higher rating than their male peers when completing a questionnaire on math anxiety, which might explain the appearance of gender differences in studies that are based on this type of instrument.

This result confirms the importance of complementing quantitative studies with a qualitative phase, which may help understand the nature and characteristics of students' affective responses. To effectively carry out this combination, there is a need for qualitative instruments that are sensitive to obstacles associated to the study of affect, a delicate issue.

It is essential that the interviewee does not feel self-conscious and is able to express his/her feelings. The inclusion of a vignette in the interview has proved to be a facilitating element when gathering information. We feel that this is a step in the right direction, opening the door to a new way of communication in the study of affect that goes from direct questioning to the approach of the problem through an unconnected situation which is attractive to the interviewee and breaks down the barrier that might exist when talking about personal feelings. 